\title{
Loosening of Implant Not Related to Bone-Ingrowth
}

National Cancer Institute

\section{Source}

National Cancer Institute. Loosening of Implant Not Related to Bone-Ingrowth. NCI

Thesaurus. Code C133497.

Problem associated with the loss of direct anchorage of an implanted device over time or due to an injury. 